\title{
SERVIÇOS COMPRADOS EM ESPECIALIDADES, EXAMES DE MÉDIA E ALTA COMPLEXIDADES: UM ESTUDO DOS GASTOS PÚBLICOS E DEMANDAS REPRIMIDAS NO MUNICÍPIO DE BENTO GONÇALVES - RS
}

\author{
SERVICES PURCHASED IN SPECIAL TIES, MEDICAL EXAMINATIONS AND HIGH \\ COMPLEXITY: A STUDY OF PUBLIC EXPENDITURE AND REQUIRED DEMANDS IN \\ BENTO GONÇALVES - RS
}

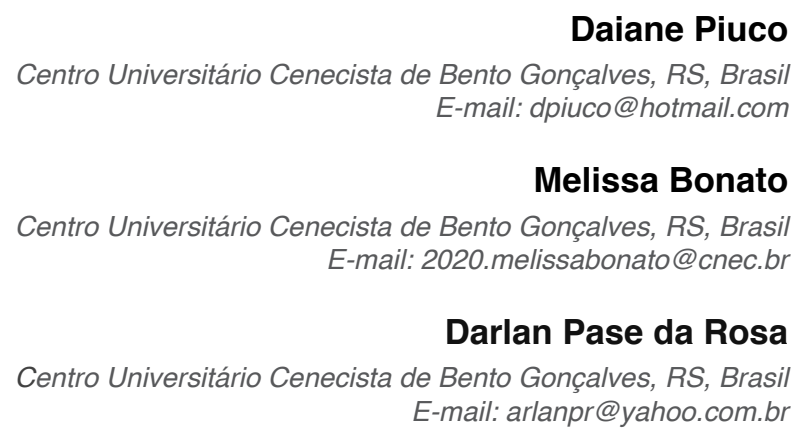

Recebido em: 18.02.2020 - Aceito em: 20. 04.2020

DOI: http://dx.doi.org/10.5902/2526629242544

RESUMO: O município de Bento Gonçalves é responsável financeiro e administrativo pela saúde pública local, a começar $p$ atenção básica, média e alta complexidade, usufruindo de recursos do Ministério da Saúde, Governo do Estado e recursos próprios. O objetivo geral do estudo foi avaliar os gastos com especialidades e exames de média e alta complexidade oferecidos aos pacientes SUS, através de serviços comprados. Trata-se de um estudo descritivo, transversal, com abordagem quantitativa. A análise realizou-se no período de outubro de 2018 até setembro de 2019, por meio da tabulação de dados obtida do sistema G-MUS e demandas reprimidas no município. Os resultados evidenciaram que os gastos mensais com especialistas chegam a $\mathrm{R} \$ 167.286 .24$, sendo que deste valor, os exames que mais geram custos são as ressonâncias magnéticas em $30 \%$, seguindo para ecocardiograma bidimensional em $11 \%$ e em terceiro lugar estão as colonoscopias e endoscopias em $10 \%$ do valor total. Através dos dados analisados, os gestores poderão utilizar os recursos com um melhor aproveitamento, reduzindo custos e demandas reprimidas.

Palavras-chave: Gestão de Serviços de Saúde; Gestão Pública; Serviços Terceirizados; Exames médicos. 
ABSTRACT: The municipality of Bento Gonçalves, it is responsible financially and administratively for local public health, from primary, medium and high complexity, with resources from the Ministry of Health, State Government and own resources. The general objective of the study was toevaluatethe expenses with specialties and medium and high complexity exams offered to SUS patients through purchased services. This is a descriptive cross-sectional study with a quantitative approach. The analysis was performed from October 2018 to September 2019, by tabulating data obtained from the G-MUS system and repressed demands in the municipality. The results showed the monthly expenseswith specialists reach $R \$ 167,286.24$, of which the most costly exams are magnetic resonances at $30 \%$, followed by two-dimensional echocardiography at $11 \%$ and third are colonoscopies and endoscopies at $10 \%$ of the total amount. Through the data analyzed, managers will be able to use resources to better use, reducing costs and repressed demands.

Keywords: Health Services Management; Public Management; Third party services; Medical exams.

\section{INTRODUÇÃO}

O Sistema Único de Saúde (SUS) foi instituído em 1988 pela Constituição Federal e regulamentado pela Lei $n^{\circ} 8080$, de 19 de setembro de 1990. Segundo esta, são consideradas atribuições, tanto do gestor estadual, como do gestor municipal, a gestão e execução de serviços públicos de atendimento à saúde da população (Brasil, 1990). Para Fonseca e Ferreira (2009), a saúde pública, resultante da combinação de diversos fatores, dentre eles os econômicos, sociais, culturais e políticos, é um dos direitos fundamentais do ser humano.

A globalização e o atual cenário político-econômico do país acabaram por impulsionar o setor de saúde a procurar novas alternativas para a gestão. A necessidade de garantir resultados positivos, mantendo a população satisfeita num mercado em permanente evolução, em que tecnologias estão cada vez mais acessíveis, requer mais que bons produtos e serviços, requer qualidade na forma de atuar (Paim \& Ciconelli, 2007). A administração desse sistema exige que ocorra um constante monitoramento e avaliação dessas ações, alimentada por informações adequadas, facilitando a tomada de decisão, minimizando erros, oferecendo serviços de qualidade (Sala, Nemes \& Cohen, 1998) e controle dos custos, pois trabalha-se com uma insuficiência de recursos para a saúde, os quais são aplicados incorretamente 
e distribuídos geograficamente de forma desigual (Rouquayrol, 2003). Isso leva a conclusão de que existe uma necessidade progressiva da implantação de atividades de controle e auditoria nos serviços de assistência à saúde.

Exames complementares são meios utilizados pelos médicos para confirmar hipóteses diagnósticas. Para Yeh (2014), o excesso de exames pode ter sua motivação em questões que envolvem desde a demanda do paciente atendido, até incentivos financeiros relacionados a essa prática. A utilização injustificada de exames pode gerar efeitos negativos, tanto para o cuidado com o paciente, quanto para a instituição, no que diz respeito ao sobrediagnóstico, que implica no diagnóstico de uma doença que nunca provocará sintomas ou a morte de um paciente, sendo um problema de saúde pública, já que converte as pessoas em pacientes sem necessidade, e leva a que sejam feitos tratamentos sem qualquer benefício em termos de saúde (Dentillo, 2012; Santos \& Toscas, 2015). Diagnósticos equivocados, gastos com recursos desnecessários e atraso em diagnósticos, são possíveis apenas com evidências clínicas (Capilheira \& Santos, 2006; Garcia et al., 2014).

No Brasil, com o processo de descentralização praticado pelo Governo Federal, que concedeu maior autonomia aos estados e municípios, emergiu a necessidade de otimizar os gastos municipais. De modo simultâneo, o aumento da participação popular na gestão das políticas públicas trouxe a exigência de maior qualidade nos serviços de saúde. Em questões como a transferência de capacidade decisória nas funções e recursos para os estados e para os municípios, as transformações foram percebidas em um grau mais elevado (Fonseca \& Ferreira, 2009).

Considerando a população estimada pelo Instituto Brasileiro de Geografia e Estatística (IBGE) de julho de 2018, o município de Bento Gonçalves possui aproximadamente 119.049 habitantes. Conforme o Plano Municipal de Saúde de 2018/2021 (Lei 8080/90) dispõe em seus artigos 7 e $8^{\circ}$, inciso I, a organização dos serviços de saúde à população é dividida em três níveis de complexidade: atenção básica ou baixa complexidade, média complexidade e alta complexidade, sendo que em cada nível os atendimentos são específicos. A atenção básica, ou baixa complexidade, através da portaria 2.488 de 21 de outubro de 2011, caracteriza-se por um conjunto de ações de saúde de promoção, proteção, prevenção, diagnóstico, tratamento, reabilitação, redução de danos e manutenção da saúde. Esta deve ser a porta de entrada dos usuários no Sistema Único de Saúde (SUS). Em Bento Gonçalves, a atenção básica conta com 11 unidades de Estratégia Saúde da Família (ESF) e 22 Unidades Básicas de Saúde (UBSs) distribuídas no município. Contam ainda com os programas: Saúde da Criança e Adolescente, Acompanha 
Bebê, Visita Bebê, Saúde da Escola, Teste do Pezinho, Saúde do Idoso, Saúde da Mulher, Saúde do Homem, Melhor em Casa, Saúde Bucal, Doenças Crônicas Não-Transmissíveis e Programas de Nutrição.

A média complexidade é composta por serviços especializados na rede, como: Saúde Mental, Centro de Referência Materno Infantil, Bloco Cirúrgico, SAE CTA (Serviço de Atendimento Especializado e Centro de Testagem e Aconselhamento), Centro Municipal de Fisioterapia, Serviço de Urgência e Emergência, SAMU Básico e Avançado, Unidade de Pronto Atendimento UPA III, Pronto Atendimento Zona Norte e Serviços de Apoio e Diagnóstico, como o Laboratório da Secretaria Municipal de Saúde, Setor de Raio X e Unidade de Saúde Central, onde são realizados exames de eletrocardiograma e espirometria sem laudo, além dos serviços comprados com especialistas. Por sua vez, na alta complexidade ou atenção terciária se enquadram os casos graves: internações, cirurgias, partos de risco, especialidades e assistência em UTI. As especialidades contratadas com o Hospital Tacchini em alta complexidade são: Oncologia, SAMUSALVAR, Gestantes de Alto Risco e Saúde Mental.

Agestão pública do município de Bento Gonçalves utiliza a terceirização para a realização dos seus serviços de média complexidade e, em alguns casos, em procedimentos de alta complexidade, gerados na atenção básica e especializada, cujos valores investidos mensalmente são de aproximadamente $R \$ 600.000,00$. Entende-se que, para que os recursos aplicados sejam bem aproveitados, torna-se necessário um estudo e controle, a fim de que possam ser oferecidos à população serviços com qualidade, eficiência, eficácia e profissionais que, além de resolverem os problemas dos pacientes, não prejudiquem o orçamento, gerando exames desnecessários.

No município de Bento Gonçalves, a maioria dos serviços especializados e exames de média e alta complexidade são comprados em prestadores conveniados, por meio de edital de chamamento público 004/2018. Dessa forma, devido ao grande volume de serviços comprados, torna-se necessário um acompanhamento, a fim de avaliar os custos e a resolução dos diagnósticos e tratamentos aos pacientes.

Com o intuito de melhoria nos encaminhamentos realizados e agendados aos especialistas, bem como a redução de custos com encaminhamentos desnecessários, desde janeiro de 2019, o município de Bento Gonçalves passou a ter um médico regulador atuando na equipe de regulação. $O$ profissional regulador atuou por 14 anos em unidades de estratégia de saúde da família do município, tendo grande experiência quanto à necessidade de exames e encaminhamentos. Em abril de 2019 foram criados e implantados, juntamente com a equipe de re- 
gulação, protocolos com critérios para solicitações de consultas especializadas e alguns exames. Os encaminhamentos recebidos das unidades passaram a ser filtrados e melhor direcionados antes do agendamento, sendo muitas vezes devolvidos para que fossem atendidos na própria atenção primária. A partir disso, as filas de espera começaram a diminuir e os serviços comprados passaram a ter uma qualidade melhor no encaminhamento.

O presente trabalho se justifica por tratar de um tema atual, onde a gestão pública utiliza a terceirização para a realização dos seus serviços gerados na atenção básica. Justifica-se também pelo estudo da efetividade aplicada aos serviços, como medida de resultado, que pode trazer benefícios aos usuários e ao município, além de contribuir para tomada de decisão de outras cidades que trabalham ou pretendem trabalhar com a terceirização de serviços na gestão da saúde.

Diante do cenário exposto, o propósito da pesquisa desenvolvida neste trabalho é analisar as demandas reprimidas e diagnosticar a condição atual dos gastos públicos por profissional, especialidade e exames gerados, abordando a questão do desperdício, uso racional de encaminhamentos, e, por fim, contribuir para uma melhor tomada de decisões na disponibilização de recursos. Através de dados obtidos com os setores de regulação e auditoria da Secretaria Municipal de Saúde, vislumbrou-se a necessidade de realizar um estudo sobre a gestão em saúde, visando otimizar os recursos disponíveis, melhorando a qualidade e resolutividade dos serviços oferecidos aos usuários.

Considerando o descrito, o objetivo geral deste trabalho foi avaliar os gastos com especialidades e exames de média e alta complexidade oferecidos aos pacientes do SUS, por meio de serviços comprados.

\section{REFERENCIAL TEÓRICO}

A porta de entrada de qualquer paciente no SUS é a Unidade Básica de Saúde (UBS) e as urgências através da Unidade de Pronto Atendimento (UPA). $\mathrm{Na}$ maioria dos casos, são encaminhados para especialistas, sendo que dificilmente os pacientes crônicos têm alta, recebendo seu tratamento por completo nos serviços especializados e gerando altos custos, caso não existam profissionais conscientes na solicitação dos exames. A perda da capacidade de resolver as principais demandas sobrecarrega os níveis de atenção secundária e terciária, gerando exames muitas vezes desnecessários e, principalmente, prejudicando os pacientes na resolução de seus problemas, criando filas de espera que, em muitos casos, fazem com que o quadro apresentado possa evoluir, comprometendo 
por vezes a possibilidade de cura do paciente ou gerando um custo muito maior (Plano Municipal de Saúde, 2018-2021).

Algumas causas como: a supervalorização dos exames, em detrimento do exame físico ou história clínica, postura médica defensiva, insegurança ou inexperiência profissional, redução do tempo da consulta médica, influência dos meios de comunicação e conflitos de interesse, desconhecimento do custo dos procedimentos e a falta de comunicação/integração entre os serviços de saúde, têm contribuído para o crescimento da demanda por exames complementares que auxiliam o diagnóstico e tratamento clínico (Aguiar, 2013). Existem diversas razões para alta solicitação de exames e encaminhamentos especializados, entretanto, antes do pedido de determinados exames, alguns requisitos são necessários para essa análise, como, por exemplo, a construção de uma rede clínica articulada, a definição de protocolos e critérios de escolha, consulta a outros profissionais de saúde e uso da medicina baseada em evidências (Leite \& Sá, 2002; Prat et al., 2009; Issa et al., 2011).

Mesmo nos países mais ricos, não existe disponibilidade de recursos financeiros suficientes para oferecer saúde de qualidade para toda a população. Portela e Teixeira (2011) identificam o planejamento e a gestão em saúde intimamente relacionados, existindo uma coesão entre o planejar e o gerir. Os mesmos autores apontam como fatores relacionados ao ato de gerir, além do planejar, a tomada de decisões, o uso da contabilidade e do financiamento em saúde, utilizando os recursos com eficiência. Tais atividades envolvem o orçamento público, as necessidades de saúde, os recursos disponíveis, com objetivo de assegurar acesso da população às tecnologias efetivas e seguras em condições de equidade (Brasil, 2010).

Exames são ferramentas de apoio ao diagnóstico médico desde a Grécia antiga. De lá para cá, houve muitas mudanças e avanços no mundo, na medicina e nos pacientes. Atualmente, é frequente o paciente sair do consultório carregado de pedidos de exames após uma consulta, a qual acontece de forma cada vez mais rápida, superficial e muitas vezes, insatisfatória. Carpilheira e Santos (2006) e dados nacionais do Sistema Único de Saúde (SUS) mostraram que no Brasil, de 52 a $76 \%$ das consultas médicas culminam no pedido de exames. Uma consulta normal prevê que o médico faça a anamnese e o exame clínico. São essas informações, juntamente com o motivo do encaminhamento, que levam o profissional a elaborar o diagnóstico, que será confirmado por exames complementares.

De acordo com Caprara e Franco (1999), para que exista e prevaleça a confiança entre médico-paciente, é necessário que o médico preencha vários requisitos, como: empatia, sinceridade, competência e sensibilidade em relação ao problema vivenciado pelo paciente. Deve-se analisar não somente a doença, mas 
também a experiência e o ponto de vista do doente e dos familiares, o contexto e histórico familiar e sua influência sobre a prevenção, o diagnóstico e o tratamento.

As causas de altos números de solicitações de exames são multifatoriais. Passam pela formação e educação dos médicos, conscientização dos pacientes, qualidade e tempo da consulta médica, remuneração dos serviços comprados, focando no impacto na saúde do paciente e não na quantidade de procedimentos solicitados. O profissional não deve se limitar a solicitar menos exames, porém os mesmos devem ser solicitados adequadamente e que garantam um melhor cuidado com a saúde do paciente (Gomes et al., 2012).

\section{MÉTODOS DE PESQUISA}

Trata-se de um estudo transversal descritivo. As variáveis utilizadas neste estudo são: os principais tipos de exames solicitados pelos especialistas, custo por profissional e especialidade, e demandas reprimidas no período de 12 meses. Como fonte de dados, foi utilizado o sistema de gestão municipal G-MUS, através dos relatórios quantitativos e financeiros dos exames complementares, solicitados pelos especialistas que possuem vínculo com o município e realizam atendimento através de serviços comprados. Foi analisada a faixa de 12 meses (outubro de 2018 até setembro de 2019), período que houve a alteração dos valores de serviços comprados, por meio de editais de chamamento público. A análise foi realizada através de relatórios gerenciais físicos e financeiros, obtidos do sistema G-MUS, utilizado pelo município para as autorizações, atendimentos e orçamentos. As variáveis utilizadas e comparadas neste estudo foram: o custo por especialidade, custo por profissional e demandas reprimidas na área.

Os dados coletados através de relatórios do sistema G-MUS, utilizado na Secretaria Municipal de Saúde de Bento Gonçalves, para controle e autorização dos serviços no período de outubro de 2018 até setembro de 2019, indicaram que foram solicitados exames de média e alta complexidade pelos especialistas que atendem através de serviços comprados pelo edital de chamamento público 004/2018 e pagos através de recurso próprio do município. Os serviços são pagos após autorização prévia do setor de regulação e conferência realizada pelo setor de auditoria, onde os prestadores entregam a comprovação do comparecimento nas consultas e os laudos da realização dos exames que foram liberados.

Os dados foram tabulados na planilha eletrônica Microsoft Office Excel® 2016 e expressos por meio de tabelas, sendo tratados através de estatística descritiva básica, utilizando a média de custo. A coleta de dados foi realizada após 
a aprovação do Comitê de Ética em Pesquisa do Centro Universitário Cenecista de Bento Gonçalves, sob o parecer n ${ }^{\circ}$ 3.511.213 e após aprovação do atual gestor da Secretaria Municipal de Saúde. Quanto aos aspectos éticos, foram atendidas as considerações da resolução n 466/2012 do Conselho Nacional de Saúde (CNS) (substituída pela Norma Operacional 001/2013), a qual trata das diretrizes e normas regulamentadoras de pesquisas, que se referem ao envolvimento de seres humanos. Da mesma forma, atendeu-se as considerações da resolução $n^{\circ}$ 510/2016 do CNS. A fim de garantir sigilo e não-identificação dos profissionais nas tabelas, substituiu-se os nomes dos especialistas por especialista 1, especialista 2 e assim sucessivamente.

\section{APRESENTAÇÃO E DISCUSSÃO DOS RESULTADOS}

Após analisar mensalmente os exames gerados pelos especialistas, utilizando o filtro por profissional solicitante, foi realizada a tabulação dos dados e, tendo em vista que alguns profissionais entraram em períodos após o período inicial, a tabela foi simplificada para que se pudesse ter o valor médio durante os 12 meses. As tabelas foram separadas em custo por profissional (Tabela 1), custo por especialidade (Tabela 2), exames mais gerados (Tabela 3 ) e demandas reprimidas no período analisado (Tabelas 4 e 5).

Tabela 1 - Custo por profissional (out/18 até set/19)

\begin{tabular}{cccc}
\hline Profissional & Consultas & Valor Gerado & Custo Médio \\
\hline Cardiologista 1 & 375 & $\mathrm{R} \$ 84.203,38$ & $\mathrm{R} \$ 224,54$ \\
\hline Cardiologista 2 & 179 & $\mathrm{R} \$ 37.713,72$ & $\mathrm{R} \$ 210,69$ \\
\hline Cardiologista 3 & 274 & $\mathrm{R} \$ 111.068,98$ & $\mathrm{R} \$ 405,36$ \\
\hline Cardiologista 4 & 165 & $\mathrm{R} \$ 75.627,05$ & $\mathrm{R} \$ 458,34$ \\
\hline Cardiologista 5 & 528 & $\mathrm{R} \$ 105.659,97$ & $\mathrm{R} \$ 200,11$ \\
\hline Cardiologista 5 & 912 & $\mathrm{R} \$ 231.987,32$ & $\mathrm{R} \$ 254,37$ \\
\hline Cardiologista 7 & 804 & $\mathrm{R} \$ 163.938,90$ & $\mathrm{R} \$ 203,90$ \\
\hline Cirurgião Geral & 94 & $\mathrm{R} \$ 8.460,00$ & $\mathrm{R} \$ 90,00$ \\
\hline Cirurgião Pediátrico & 93 & $\mathrm{R} \$ 10.977,76$ & $\mathrm{R} \$ 118,04$ \\
\hline Cirurgião Vascular 1 & 340 & $\mathrm{R} \$ 55.220,00$ & $\mathrm{R} \$ 162,41$ \\
\hline Cirurgião Vascular 2 & 713 & $\mathrm{R} \$ 130.470,00$ & $\mathrm{R} \$ 182,98$ \\
\hline Cirurgião Vascular 3 & 480 & $\mathrm{R} \$ 46.950,00$ & $\mathrm{R} \$ 97,81$ \\
\hline Cirurgião Vascular 4 & 258 & $\mathrm{R} \$ 34.174,84$ & $\mathrm{R} \$ 132,46$ \\
\hline Dermatologista & 239 & $\mathrm{R} \$ 21.870,00$ & $\mathrm{R} \$ 91,50$ \\
\hline
\end{tabular}




\begin{tabular}{|c|c|c|c|}
\hline Endocrinologista 1 & 178 & $\mathrm{R} \$ 20.127,72$ & $\mathrm{R} \$ 113,07$ \\
\hline Endocrinologista 2 & 515 & $\mathrm{R} \$ 59.213,95$ & $\mathrm{R} \$ 114,97$ \\
\hline Gastroenterologista & 760 & $\mathrm{R} \$ 393.000,68$ & $\mathrm{R} \$ 517,10$ \\
\hline Hematologista 1 & 361 & $\mathrm{R} \$ 41.785,53$ & $\mathrm{R} \$ 115,74$ \\
\hline Hematologista 2 & 212 & $\mathrm{R} \$ 21.720,00$ & $\mathrm{R} \$ 102,45$ \\
\hline Mastologista & 29 & $\mathrm{R} \$ 2.750,00$ & $\mathrm{R} \$ 97,82$ \\
\hline Neurologista 1 & 852 & $\mathrm{R} \$ 145.012,21$ & $\mathrm{R} \$ 170,20$ \\
\hline Neurologista 2 & 522 & $\mathrm{R} \$ 74.174,97$ & $\mathrm{R} \$ 142,09$ \\
\hline Neurologista 3 & 545 & $\mathrm{R} \$ 143.410,15$ & $\mathrm{R} \$ 263,13$ \\
\hline Neurologista 4 & 684 & $\mathrm{R} \$ 126.905,24$ & $\mathrm{R} \$ 185,53$ \\
\hline Ortopedista 1 & 888 & $\mathrm{R} \$ 139.143,77$ & $\mathrm{R} \$ 156,69$ \\
\hline Ortopedista 2 & 113 & $\mathrm{R} \$ 19.911,10$ & $\mathrm{R} \$ 176,20$ \\
\hline Ortopedista 3 & 560 & $\mathrm{R} \$ 115.058,75$ & $\mathrm{R} \$ 205,46$ \\
\hline Ortopedista 4 & 293 & $\mathrm{R} \$ 36.816,32$ & $\mathrm{R} \$ 125,65$ \\
\hline Ortopedista 5 & 296 & $\mathrm{R} \$ 62.783,12$ & $\mathrm{R} \$ 212,10$ \\
\hline Ortopedista 6 & 192 & $\mathrm{R} \$ 21.744,25$ & $\mathrm{R} \$ 113,25$ \\
\hline Ortopedista 7 & 1605 & $\mathrm{R} \$ 385.131,60$ & $\mathrm{R} \$ 239,95$ \\
\hline Ortopedista 8 & 1488 & $\mathrm{R} \$ 283.281,68$ & $\mathrm{R} \$ 190,37$ \\
\hline Otorrinolaringologista 1 & 361 & $\mathrm{R} \$ 60.415,81$ & $\mathrm{R} \$ 167,35$ \\
\hline Otorrinolaringologista 2 & 513 & $\mathrm{R} \$ 62.262,54$ & $\mathrm{R} \$ 121,36$ \\
\hline Otorrinolaringologista 3 & 421 & $\mathrm{R} \$ 63.000,56$ & $\mathrm{R} \$ 149,64$ \\
\hline Pneumologista 1 & 588 & $\mathrm{R} \$ 74.134,36$ & $\mathrm{R} \$ 126,07$ \\
\hline Pneumologista 2 & 573 & $\mathrm{R} \$ 62.062,07$ & $\mathrm{R} \$ 108,31$ \\
\hline Proctologista 1 & 162 & $\mathrm{R} \$ 15.710,00$ & $\mathrm{R} \$ 96,97$ \\
\hline Proctologista 2 & 331 & $\mathrm{R} \$ 125.371,67$ & $\mathrm{R} \$ 378,76$ \\
\hline Reumatologista & 739 & $\mathrm{R} \$ 77.263,44$ & $\mathrm{R} \$ 104,55$ \\
\hline Urologista 1 & 797 & $\mathrm{R} \$ 101.721,18$ & $\mathrm{R} \$ 127,63$ \\
\hline Urologista 2 & 221 & $\mathrm{R} \$ 28.322,85$ & $\mathrm{R} \$ 128,15$ \\
\hline Urologista 3 & 36 & $\mathrm{R} \$ 3.796,07$ & $\mathrm{R} \$ 105,44$ \\
\hline Urologista 4 & 43 & $\mathrm{R} \$ 5.179,52$ & $\mathrm{R} \$ 120,45$ \\
\hline Total & 20332 & $\mathrm{R} \$ 3.889 .533,03$ & R\$ 191,30 \\
\hline
\end{tabular}

Fonte: Elaboração própria.

Entre os 7 cardiologistas, verifica-se que o cardiologista 4 seguido pelo cardiologista 3 são os que apresentam um custo mais elevado. O cardiologista 5 custa a metade do valor dos cardiologistas 3 e 4 . Nas demais especialidades, em que há mais de um profissional atendendo, também é possível ver a diferença de custo entre os profissionais. 
Tabela 2 - Custo por especialidade (out/2018 até set/2019)

\begin{tabular}{cccc}
\hline Especialidade & Consultas & Valor Gerado & Custo Médio \\
\hline Cardiologista & 3237 & $\mathrm{R} \$ 810.199,32$ & $\mathrm{R} \$ 250,29$ \\
\hline Cirurgião Geral & 94 & $\mathrm{R} \$ 8.460,00$ & $\mathrm{R} \$ 90,00$ \\
\hline Cirurgião Pediátrico & 93 & $\mathrm{R} \$ 10.977,76$ & $\mathrm{R} \$ 118,04$ \\
\hline Cirurgião Vascular & 1791 & $\mathrm{R} \$ 266.814,84$ & $\mathrm{R} \$ 148,97$ \\
\hline Dermatologista & 239 & $\mathrm{R} \$ 21.870,00$ & $\mathrm{R} \$ 91,50$ \\
\hline Endocrinologista & 693 & $\mathrm{R} \$ 79.341,67$ & $\mathrm{R} \$ 114,49$ \\
\hline Gastroenterologista & 760 & $\mathrm{R} \$ 393.000,68$ & $\mathrm{R} \$ 517,10$ \\
\hline Hematologista & 573 & $\mathrm{R} \$ 63.505,53$ & $\mathrm{R} \$ 110,83$ \\
\hline Mastologista & 29 & $\mathrm{R} \$ 2.750,00$ & $\mathrm{R} \$ 94,82$ \\
\hline Neurologista & 2603 & $\mathrm{R} \$ 489.502,57$ & $\mathrm{R} \$ 188,05$ \\
\hline Ortopedista & 5435 & $\mathrm{R} \$ 1.063 .870,59$ & $\mathrm{R} \$ 195,74$ \\
\hline Otorrinolaringologista & 1295 & $\mathrm{R} \$ 185.678,91$ & $\mathrm{R} \$ 143,38$ \\
\hline Pneumologista & 1161 & $\mathrm{R} \$ 136.196,43$ & $\mathrm{R} \$ 117,30$ \\
\hline Proctologista & 493 & $\mathrm{R} \$ 141.081,67$ & $\mathrm{R} \$ 286,17$ \\
\hline Reumatologista & 739 & $\mathrm{R} \$ 77.263,44$ & $\mathrm{R} \$ 104,55$ \\
\hline Urologista & 1097 & $\mathrm{R} \$ 139.019,62$ & $\mathrm{R} \$ 126,73$ \\
\hline Total & $\mathbf{2 0 3 3 2}$ & $\mathrm{R} \$ \mathbf{3 . 8 8 9 . 5 3 3 , 0 3}$ & $\mathrm{R} \$ 191,30$ \\
\hline
\end{tabular}

Fonte: Elaboração própria.

Na tabela 2, foi realizada a média de custo por especialidade dentro do período de 12 meses. Entre as especialidades, ortopedia, cardiologia e neurologia foram as que mais tiveram consultas compradas. Atualmente, o valor pago por cada consulta especializada é de $\mathrm{R} \$ 90,00$ do qual a Secretaria Municipal de Saúde realizou a média de custo através de orçamentos e lançou em outubro de 2018 um edital de chamamento público, cujos profissionais interessados realizaram o credenciamento.

Tabela 3 - Exames mais solicitados

\begin{tabular}{ccc}
\hline Procedimentos Mais Solicitados & Físico & Financeiro \\
\hline Ressonância Magnética com ou sem Contraste & 1984 & $\mathrm{R} \$ 610.180,50$ \\
\hline Ultrassonografia sem Doppler & 1462 & $\mathrm{R} \$ 102.340,00$ \\
\hline Ultrassonografia com Doppler & 1248 & $\mathrm{R} \$ 112.320,00$ \\
\hline Ecocardiograma Bidimensional com ou sem Doppler & 977 & $\mathrm{R} \$ 212.497,50$ \\
\hline Tomografias com ou sem Contraste & 859 & $\mathrm{R} \$ 143.174,36$ \\
\hline Eletroencefalograma Solo e Vigília & 577 & $\mathrm{R} \$ 47.008,10$ \\
\hline Endoscopia Digestiva Alta & 365 & $\mathrm{R} \$ 191.536,51$ \\
\hline Holter 24 Horas & 356 & $\mathrm{R} \$ 58.683,04$ \\
\hline Colonoscopias & 286 & $\mathrm{R} \$ 216.723,48$ \\
\hline Cintilografias & 252 & $\mathrm{R} \$ 117.052,19$ \\
\hline & &
\end{tabular}




\begin{tabular}{ccc}
\hline Teste Ergométrico & 240 & $\mathrm{R} \$ 34.580,00$ \\
\hline Ecocardiograma com Stress Farmacológico & 133 & $\mathrm{R} \$ 73.482,92$ \\
\hline Mapa 24 Horas & 116 & $\mathrm{R} \$ 19.121,44$ \\
\hline Videolaringoscopia & 93 & $\mathrm{R} \$ 14.925,06$ \\
\hline Endoscopia Nasal & 84 & $\mathrm{R} \$ 13.370,28$ \\
\hline Eletroneuromiografia Por Membro & 72 & $\mathrm{R} \$ 19.183,92$ \\
\hline Biópsias & 44 & $\mathrm{R} \$ 16.060,00$ \\
\hline Eletrocardiograma com Laudo & 26 & $\mathrm{R} \$ 853,06$ \\
\hline Uretrocistografia & 14 & $\mathrm{R} \$ 1.429,54$ \\
\hline RX Contrastado & 8 & $\mathrm{R} \$ 719,54$ \\
\hline Ecocardiograma Transesofágico/Transtorácico & 4 & $\mathrm{R} \$ 2.193,52$ \\
\hline
\end{tabular}

Fonte: Elaboração própria.

Analisando os exames gerados pelos especialistas no período, verifica-se que ressonância magnética, ultrassonografia, ecocardiograma bidimensional e tomografia estão entre os mais solicitados. Em termos de exames que mais geram impacto no orçamento, o primeiro lugar permanece com as ressonâncias magnéticas com $30 \%$, seguindo para ecocardiogramas bidimensionais (com e sem doppler) com $11 \%$ e, em terceiro lugar, com $10 \%$, estão as colonoscopias e endoscopias.

Além dos riscos à saúde física dos pacientes, o alto índice de solicitações de exames de imagem, muitas vezes desnecessários, oneram a saúde financeira do município, devido ao alto custo dos procedimentos. Chama atenção o caso das ressonâncias magnéticas. Segundo Reinaldo de Camargo Scheibe, presidente da Associação Brasileira de Planos de Saúde, entre os brasileiros com plano privado são feitos, a cada ano, 148 exames para cada grupo de 1.000 pessoas. É um número ligeiramente superior até mesmo ao dos exagerados americanos e vergonhoso quando comparado ao de outros países.

Na Austrália, por exemplo, são feitos 42 exames para cada 1.000 habitantes. No Canadá, cerca de 56. Exames mais sofisticados são uma importante fonte de receita financeira, tanto para médicos, como para hospitais. Dados nacionais do SUS mostraram que, no Brasil, de 52 a $76 \%$ das consultas culminam no pedido de exames (OPS, 2002). Uma hipótese seria que os exames estariam sendo pedidos, em boa parte, para os mesmos indivíduos em locais diferentes. Segundo Bates DW, a repetição de exames já foi apontada por estudo norte americano, que encontrou uma prevalência de $28 \%$ de exames repetidos antes do intervalo de tempo preconizado, em pacientes hospitalizados. Um estudo holandês citado por Branger (1995) encontrou prevalência de 38\% de repetição de exames no período de oito meses, em pacientes ambulatoriais. Aproximadamente $35 \%$ dos exames repetidos 
foram solicitados por médicos diferentes dos que fizeram o pedido inicial.

Outra possibilidade é que alguns pacientes precisam consultar em mais de um local, de forma a obter a solicitação de algum exame complementar. Através dessa análise, pode-se ter uma programação financeira de quais exames necessitam de um maior investimento para que não sejam criadas demandas reprimidas.

Tabela 4 - Demanda de especialidades compradas (primeira consulta) na regulação

\begin{tabular}{|c|c|c|c|c|c|c|c|c|c|c|c|c|}
\hline \multirow{2}{*}{ Especialidade } & Out & Nov & Dez & Jan & Fev & Mar & Abr & Mai & Jun & Jul & Ago & Set \\
\hline & 18 & 18 & 18 & 19 & 19 & 19 & 19 & 19 & 19 & 19 & 19 & 19 \\
\hline Cardiologia & 344 & 443 & 505 & 419 & 359 & 254 & 0 & 0 & 0 & 0 & 0 & 0 \\
\hline Cirur & 0 & 0 & 0 & 0 & 0 & 0 & 66 & 16 & 103 & 205 & 250 & 180 \\
\hline Cirurgia vascular & 1159 & 1150 & 863 & 638 & 529 & 322 & 0 & 0 & 0 & 0 & 0 & 0 \\
\hline Derma & 339 & 268 & 316 & 156 & 0 & 0 & 0 & 0 & 0 & 0 & 0 & 0 \\
\hline Endocrinologia & 71 & 71 & 98 & 70 & 111 & 131 & 105 & 126 & 272 & 257 & 217 & 326 \\
\hline $\begin{array}{c}\text { Gastroenterologia } \\
\text { adulto }\end{array}$ & 477 & 483 & 597 & 565 & 569 & 600 & 593 & 284 & 206 & 123 & 79 & 80 \\
\hline Hematologia & 70 & 66 & 109 & 73 & 58 & 64 & 50 & 37 & 0 & 0 & 0 & 0 \\
\hline Masto & 8 & 8 & 9 & 10 & 23 & 30 & 35 & 27 & 13 & 0 & 0 & 0 \\
\hline Neurologia & 646 & 626 & 642 & 582 & 239 & 0 & 0 & 0 & 0 & 0 & 0 & 0 \\
\hline Ortopedia & 177 & 359 & 454 & 512 & 377 & 400 & 473 & 618 & 225 & 0 & 0 & 0 \\
\hline Otorrinolaringologia & 0 & 61 & 454 & 85 & 0 & 0 & 0 & 0 & 0 & 0 & 0 & 0 \\
\hline Pneumologia & 7 & 2 & 35 & 0 & 89 & 57 & 0 & 0 & 0 & 0 & 0 & 0 \\
\hline Proctologia & 190 & 261 & 259 & 229 & 244 & 242 & 232 & 0 & 0 & 0 & 0 & 0 \\
\hline Reumatologia & 0 & 0 & 23 & 31 & 36 & 12 & 0 & 0 & 0 & 0 & 0 & 0 \\
\hline Urologia & 253 & 271 & 188 & 10 & 0 & 0 & 0 & 34 & 0 & 0 & 0 & 0 \\
\hline
\end{tabular}

Fonte: Elaboração própria.

Nas Tabelas 4 e 5, pode-se acompanhar as demandas reprimidas em primeiras consultas e exames no período estudado. As filas de espera para tratamentos especializados eletivos são uma constante preocupação, não somente para a gestão do município, mas em todos os países. Para os autores Paterson et al. (2006), o tempo de espera aceitável para a primeira consulta ao especialista 
não deve ultrapassar três meses. Como em outros municípios brasileiros, o problema da fila de espera é antigo, persistindo apesar da troca de equipes de gestão da saúde.

Tabela 5 - Demanda de exames no período estudado

\begin{tabular}{|c|c|c|c|c|c|c|c|c|c|c|c|c|}
\hline \multirow{2}{*}{ Exames } & Out & Nov & Dez & Jan & Fev & Mar & Abr & Mai & Jun & Jul & Ago & Set \\
\hline & 18 & 18 & 18 & 19 & 19 & 19 & 19 & 19 & 19 & 19 & 19 & 19 \\
\hline Colonoscopia & 131 & 153 & 151 & 166 & 191 & 203 & 197 & 261 & 246 & 242 & 230 & 200 \\
\hline Endoscopia digestiva alta & 195 & 236 & 213 & 218 & 243 & 257 & 197 & 371 & 311 & 293 & 264 & 271 \\
\hline Biópsia & 44 & 30 & 35 & 40 & 58 & 43 & 20 & 0 & 0 & 4 & 3 & 0 \\
\hline Cintilografia & 38 & 16 & 35 & 11 & 0 & 0 & 0 & 0 & 0 & 12 & 12 & 14 \\
\hline $\begin{array}{l}\text { Ultrassonografia com ou } \\
\text { sem Doppler }\end{array}$ & 932 & 1500 & 1000 & 500 & 50 & 149 & 0 & 0 & 0 & 0 & 0 & 0 \\
\hline $\begin{array}{c}\text { Eletroencefalograma sono / } \\
\text { vigília }\end{array}$ & 0 & 65 & 91 & 125 & 6 & 47 & 12 & 0 & 0 & 0 & 0 & 0 \\
\hline $\begin{array}{l}\text { Eletrocardiograma com } \\
\text { laudo }\end{array}$ & 0 & 500 & 800 & 800 & 400 & 0 & 0 & 0 & 0 & 0 & 0 & 0 \\
\hline $\begin{array}{l}\text { Ecocardiograma com Do- } \\
\text { ppler }\end{array}$ & 129 & 49 & 57 & 37 & 0 & 0 & 0 & 0 & 0 & 29 & 0 & 0 \\
\hline $\begin{array}{l}\text { Ecocardiograma com stress } \\
\text { farmacológico }\end{array}$ & 2 & 2 & 6 & 0 & 0 & 0 & 0 & 0 & 0 & 0 & 0 & 0 \\
\hline $\begin{array}{l}\text { Holter / mapa cardíaco } 24 \\
\text { horas }\end{array}$ & 30 & 10 & 6 & 2 & 0 & 0 & 0 & 0 & 0 & 39 & 0 & 0 \\
\hline Teste ergométrico & 73 & 28 & 35 & 9 & 0 & 0 & 0 & 0 & 0 & 3 & 0 & 0 \\
\hline Tomografia & 68 & 109 & 167 & 124 & 100 & 156 & 15 & 6 & 2 & 0 & 0 & 0 \\
\hline Ressonância magnética & 60 & 59 & 204 & 114 & 101 & 10 & 18 & 21 & 25 & 0 & 0 & 0 \\
\hline
\end{tabular}

Fonte: Elaboração própria.

No município de Bento Gonçalves, os pacientes encaminhados aos especialistas são prontamente agendados, de acordo com o quadro clínico e agendas disponíveis. O tempo de espera para as especialidades eletivas de endocrinologia, gastroenterologia e cirurgião geral, únicas especialidades atualmente reprimidas, apresentam um tempo médio de 6 meses para endocrinologia, 5 meses para gastroenterologia e 5 meses para cirurgião geral.

O tempo de espera é exclusivamente devido à pouca oferta e poucos especialistas credenciados nessas áreas. Segundo Kirwan (1997), simplesmente aumentar a oferta de consultas, visando reduzir a lista de espera, apenas aumentaria o número de encaminhamentos. A solução passa também, provavelmente, por melhor comunicação entre o médico (clínico geral ou de comunidades) e o especialista. Huber et al. (2008) afirmam que para diminuir a demanda reprimida por atendimentos eletivos é necessário que se proporcione acesso equânime aos ser- 
viços de saúde. Isso inclui a política de se proporcionar o acesso a todo cidadão a atendimento básico de alta qualidade e estabelecimento de um tempo limite para o acesso à média complexidade, caso seja necessário.

\section{CONSIDERAÇÕES FINAIS}

No município de Bento Gonçalves, grande parte dos serviços especializados e exames de média e alta complexidade são comprados em prestadores conveniados, por meio de edital de chamamento público (004/2018), sendo que os gastos mensais somente com especialistas chegam a $R \$ 167.286,24$.

O controle de custos é um conceito chave que deve ser absorvido na tentativa de reduzir orçamentos e frear o crescimento da demanda por serviços de saúde. Oferecer uma assistência à saúde custo-efetiva, frente às inovações tecnológicas, não basta. É preciso também introduzir um processo racional e, sobretudo, possuir bom senso no seu desempenho diário. Desenvolver uma medicina de qualidade e ao mesmo tempo custo-efetiva requer uma nova postura profissional. É necessário e imprescindível motivar os médicos para que se inicie a ter um controle dos gastos em saúde.

Nesse sentido, para consolidar os princípios do SUS, considerando o acesso e a integralidade da assistência, se faz necessária a integração dos serviços e saúde. Esta deve ser desenvolvida através de redes assistenciais, operando de forma articulada, por meio de uma linha de cuidado pactuada entre todos os níveis envolvidos, nas quais o sistema de referência e contra referência seja efetivo e estratégico no processo de gestão do cuidado.

Outra ação a ser adotada para o desenvolvimento de uma medicina custo-efetiva de qualidade é quantificar os exames solicitados por cada médico, fazendo com que eles entendam os impactos financeiros gerados em cada atendimento. Os resultados do estudo permitirão aos gestores diminuir a compra de consultas com os profissionais que geram mais exames de alto custo, reduzindo assim os orçamentos e demandas reprimidas, e permitindo utilizar os recursos para compra de mais serviços eficientes e de qualidade.

A maior atenção quanto aos gastos públicos em saúde, principalmente em um país em desenvolvimento como o Brasil, é de extrema relevância e precisa ser abordada nas universidades e instituições de ensino, a fim de formar profissionais mais capacitados e preparados para lidarem com as adversidades da prática.

O estudo limitou-se a abordar os serviços comprados na área da saúde, identificando as vantagens e desvantagens para a Gestão Pública do Município 
de Bento Gonçalves na resolução dos casos que não conseguem ser atendidos na atenção básica e são encaminhados à atenção especializada bem como as filas de espera para esses serviços. Faz-se relevante nova pesquisa sobre auditoria das solicitações de exames por especialidade, uma vez que surgiu a hipótese de que profissionais podem solicitar exames não fundamentais ou fora da sua especialização. Também se sugere um estudo sobre a resolutividade da atenção básica, analisando exames e encaminhamentos a serviços especializados. Elevada solicitação de exames complementares e encaminhamentos geram aumento das filas de espera, retardam os resultados mais urgentes e aumentam os custos financeiros, além de causar insatisfação entre os usuários.

\section{REFERÊNCIAS}

Aguiar, F. J. B.; Ferreira-Júnior, M.; Sales, M. M.; Cruz-Neto L. M.; Fonseca L. A. M. \& Sumita, N. M. (2013). Proteína C reativa: aplicações clínicas e propostas para utilização racional. Rev. Assoc. Med. Bras., 59(1), 85-92.

Bates, D. W.; Boyle, D. L.; Rittenberg, E.; Kuperman, G. J.; Ma'Luf, N. \& Menkin, V. (1998). What proportion of common diagnostic tests appear redundant. Am J Med., 104(4), 361-8.

Brasil. Lein ${ }^{\circ} 8.666$ de21 de junho de 1993. Regulamenta o art. 37, incisoXXI, da Constituição Federal, institui normas para licitações e contratos da Administração Públicaedáoutrasprovidências. DiárioOficialdaRepúblicaFederativadoBrasil, Brasília, DF.

Brasil. Constituição da República Federativa do Brasil de 1988. Planalto, Brasília - DF, 2013; Seção Legislação. Retirado de http://www.planalto.gov.br/ ccivil_03/constituicao/constituicaocompilado.htm.

Brasil. Ministério da Saúde. Secretaria-Executiva. Departamento de Apoio à Descentralização. Regionalização solidária e cooperativa: orientações para sua implementação no SUS. Brasília: Ministério da Saúde, 2007. 48 p. (Série A. Normas e Manuais Técnicos) (Série Pactos pela Saúde; v. 3).

Branger P. J.; Van Oers, R. J.; Van der Wouden, J. C. \& Van der Lei, J. (1995). Laboratory services utilization: a survey of repeat in investigations in ambulatory care. Neth J Med., 47(5), 208-13.

Capilheira, M. F. \& Santos, I. S. (2006). Epidemiologia da solicitação de exame complementar em consultas médicas. Revista de Saúde Pública, 40(2), 289-297. 
Caprara A \& Franco L. S. A. (1999). A relação paciente-médico. Para uma humanização da prática médica. Cadernos de Saúde Pública, 15(3), 647-654

Cardoso, F. H. (2006). Reforma do Estado. In Pereira, L. C. B. \& Spink, P. Reforma do Estado e Administração Pública Gerencial (coletânea). (7 ed). Rio de Janeiro: Editora FGV.

Castro, R. B. (2006). Eficácia, Eficiência e Efetividade na Administração Pública. $30^{\circ}$ encontro da ANPAD, Salvador.

Chiavenato, I. (1994). Recursos humanos na Empresa: pessoas, organizações e sistemas. (3 ed). São Paulo: Atlas.

Costa, F. L. \& Castanhar, J. C. (2003). Avaliação de programas públicos: desafios conceituais e metodológicos. Revista de Administração Pública, 37(5), 962-969.

Dentillo, D. B. (2012). Excesso de exames para detecção de doenças pode gerar diagnósticos prematuros e ações desnecessárias. Ciência e Cultura, 64 (3), 10-13.

Donabedian, A. (1978). The quality of medical care. Science 200, 856-864.

Fonseca, P. C. \& Ferreira, M. A. M. (2009). Investigação dos níveis de eficiência na utilização de recursos no setor de saúde: uma análise das microrregiões de Minas Gerais. Saúde e Sociedade, 18(2), 199-213.

Garcia, A. P.; Pastorio, K. A.; Nunes, R. L.; Locks, G. F. \& de Almeida, M. C. S. (2014). Indicación de exámenes preoperatorios según criterios clínicos: necesidad de supervisión. Brazilian Journal of Anesthesiology, 64(1), 54-61.

Gomes, A. M. A; Caprara, A.; Landim, L. O. P. \& Vasconcelos, M. G. F. (2012). Relação médico-paciente: entre o desejável e o possível na atenção primária à saúde. Physis: Revista de Saúde Coletiva, 22(3), 1101-1119.

Huber, M.; Stanciole, A.; Wahlbeck, K.; Tamsma, N.; Torres, F.; Jelfs, E. \& Bremner, J. (2008). Quality in and Equality of Access to Healthcare Services. Directorate-General for Employment, Social Affairs and Equal Opportunities. Luxembourg: European Communities.

Issa, M. R. N.; Isoni, N. F. C.; Soares, A. M. \& Fernandes, M. L. (2011). Avaliação pré-anestésica e redução dos custos do preparo pré-operatório. Revista Brasileira de Anestesiologia, 61(1), 65-71.

Kirwan J. (1997). Rheumatology out-patient workload increase inexorably. British J Rheumatol, 36, 481-6.

Leite, Á. J. M. \& Sá, M. (2002). Medicina Baseada em Evidências. SANARE-Revista de Políticas Públicas, 3(1). 
Nascimento, A. A. M. (2009). Regulação em Saúde: Aplicabilidade para Concretização do Pacto de Gestão do SUS. Cogitare Enferm, Curitiba, 14(2), 346-52.

Paim, Chennyfer da Rosa Paino \& Ciconelli, Rozana Mesquita. (2007). Auditoria de avaliação da qualidade dos serviços de saúde. Revista de Administração em Saúde, 9(36). Retirado de bases.bireme.br.

Paterson, W. G.; Depew, W. T.; Paré, P.; Petrunia, D.; Switzer, C.; Veldhuyzen van Zanten, S. J. \& Daniels, S. (2006). Wait times for gastroenterology consultation in Canada: The patients' perspective. Can J Gastroenterol. 24(1), 411-23.

Polonio, W. A. (2000). Terceirização: aspectos legais, trabalhistas e tributários. São Paulo: Atlas.

Portela, L. E. \& Teixeira C. F. (2001). Epidemiologia e gestão de serviços de saúde. In Barreto, M. L. \& Almeida Filho, N. Epidemiologia \& Saúde: fundamentos, métodos e aplicações Rio de Janeiro: Guanabara Koogan, pp. 622-630.

Prat, G.; Lefevre, M.; Nowak, E.; Tonnelier, J. M.; Renault, A.; L'her, E. \& Boles, J. M. (2009). Impact of clinical guidelines to improve appropriateness of laboratory tests and chest radiographs. Intensive Care Medicine, 35(6), 1047-53.

Prefeitura Municipal de Bento Gonçalves, RS. Secretaria Municipal de Saúde. Plano Municipal de Saúde. 2018-2021.

Ramos, D. M. O. (2001). Terceirização na Administração Pública. São Paulo: Ltr.

Rede Interagencial de Informações para a Saúde - RIPSA. (2002). Indicadores básicos de saúde no Brasil: conceitos e aplicações. Brasília (DF): Organização Pan-Americana de Saúde.

Rittenberg, E., Kuperman, G. J., Ma'Luf, N., \& Menkin, V. (1998). What proportion of common diagnostic tests appear redundant. Am J Med., 104(4), 361-8.

Rouquayrol, M. Z. \& Almeida Filho, N. (2003). Epidemiologia e Saúde. (6 ed). Rio de Janeiro, Medsi.

Sala, A.; Nemes, M. I. B. \& Cohen, D. D. (1998). Metodologia de avaliação do trabalho na atenção primária à saúde. Cad. Saúde Pública, Rio de Janeiro, 14(4), 741-751.

Santos T. F. \& Toscas, F. (2015). Sobrediagnóstico e suas implicações na engenharia clínica. Revista Bioética, 23(3).

Simon, J. C.; Maltchik, M.; Silva, E. E.; Lima, V. R. B. \& Bredemeier, M. (2008). Avaliação do tempo de espera para consultas de reumatologia em um centro de atendimento terciário de Porto Alegre - RS. Rev AMRIGS, 52(4), 303-8.

Zucchi, P.; Del Nero, C. \& Malik, A. M. (1998). Gastos em saúde: Os fatores que 
agem na demanda e na oferta dos serviços de saúde. Rev. Administ. Pública, 32(5), 124-47.

Yeh, D. D. (2014). A clinician's perspective on laboratory utilization management. Clinica Chimica Acta, 427, 145-50. 